\title{
Induction of G2/M phase cell cycle arrest and apoptosis by ginsenoside $R f$ in human osteosarcoma MG-63 cells through the mitochondrial pathway
}

\author{
WEN-JI SHANGGUAN ${ }^{1},{\text { HE } \mathrm{LI}^{1} \text { and YUE-HUI ZHANG }}^{2}$ \\ ${ }^{1}$ Department of Traditional Chinese Medicine, Renji Hospital, Shanghai Jiaotong University \\ School of Medicine, Shanghai 200127; ${ }^{2}$ Department of Orthopedic Surgery, Xinhua Hospital, \\ Shanghai Jiaotong University School of Medicine, Shanghai 200092, P.R. China
}

Received September 9, 2013; Accepted October 11, 2013

DOI: $10.3892 /$ or.2013.2815

\begin{abstract}
Ginsenosides, extracted from the traditional Chinese herb ginseng, are a series of novel natural anticancer products known for their favorable safety and efficacy profiles. The present study aimed to investigate the cytotoxicity of ginsenoside $\mathrm{Rf}$ to human osteosarcoma cells and to explore the anticancer molecular mechanisms of ginsenoside Rf. Five human osteosarcoma cell lines (MG-63, OS732, U-2OS, HOS and SAOS-2) were employed to investigate the cytotoxicity of ginsenoside Rf by MTT and colony forming assays. After treatment with ginsenoside Rf, MG-63 cells which were the most sensitive to ginsenoside Rf, were subjected to flow cytometry to detect cell cycle distribution and apoptosis, and nuclear morphological changes were visualized by Hoechst 33258 staining. Caspase-3, -8 and -9 activities were also evaluated. The expression of cell cycle markers including cyclin B1 and Cdk1 was detected by RT-PCR and western blotting. The expression of apoptotic genes Bcl-2 and Bax and the release of cytochrome $c$ were also examined by western blotting. Change in the mitochondrial membrane potential was observed by JC-1 staining in situ. Our results demonstrated that the cytotoxicity of ginsenoside Rf to these human osteosarcoma cell lines was dose-dependent, and the MG-63 cells were the most sensitive to exposure to ginsenoside Rf. Additionally, ginsenoside Rf induced G2/M phase cell cycle arrest and apoptosis in MG-63 cells. Furthermore, we observed upregulation of Bax and downregulation of $\mathrm{Bcl}-2, \mathrm{Cdk} 1$ and cyclin $\mathrm{B} 1$, the activation of caspase- 3 and -9 and the release of cytochrome $c$ in MG-63 cells following treatment with ginsenoside Rf. Our findings demonstrated that ginsenoside $\mathrm{Rf}$ induces $\mathrm{G} 2 / \mathrm{M}$ phase cell cycle arrest and apoptosis in human osteosarcoma MG-63
\end{abstract}

Correspondence to: Dr Yue-Hui Zhang, Department of Orthopedic Surgery, Xinhua Hospital, Shanghai Jiaotong University School of Medicine, 1665 Kongjiang Road, Shanghai 200092, P.R. China E-mail: zyhandy8030@126.com

Key words: ginsenoside Rf, human osteosarcoma, G2/M arrest, apoptosis, mitochondrial pathway cells through the mitochondrial pathway, suggesting that ginsenoside $\mathrm{Rf}$, as an effective natural product, may have a therapeutic effect on human osteosarcoma.

\section{Introduction}

Panax ginseng was discovered over 5,000 years ago in China and has since been regarded as a highly venerated medicinal herb. In recent years, natural products from plants, including ginsenosides, have attracted increased attention due to their favorable safety and efficacy profiles, suggesting a potential therapeutic application in the treatment of cancers. Recently, it has been reported that ginsenosides exhibit cytotoxic activity in a variety of human tumor cell lines (1). Previous studies revealed that ginsenoside $\operatorname{Rg} 3$ contributes to a reduction in proliferation $(2,3)$, metastasis $(3,4)$ and induction of cell death (5) in several types of cancer cells in vivo and in vitro. But few studies have focused on its effects on human osteosarcoma cell lines. Moreover, ginsenoside Rh2 has been demonstrated to have anticancer effects $(5,6)$ and to be capable of inhibiting cell growth and inducing cell cycle arrest and apoptosis in mammalian tumor cells (7). Based on these previous studies, ginseng compounds have been considered as potential agents for cancer chemotherapy.

Structurally, most of the ginsenosides belong to the protopanaxadiol (PPD), and protopanaxatriol (PPT) groups (8). Previous reports have identified that ginsenoside Rg1 and Rh1 have the most potent effect on the inhibition of cell proliferation in cancer cells $(9,10)$. In fact, steam treatment with increasing concentrations of ginsenoside Rg1 and Rh1 augments the antiproliferative activities of those ginsenosides. Ginsenoside $\mathrm{Rf}$ is a type of extract from the active constituents of ginseng, and has a structure characteristic of the presence of a hydroxyl group/sugar residue at C-6 in PPTs and is unique to Asian ginseng (1). It has been proposed that ginsenoside $\mathrm{Rf}$ may play an important role in regulating cancer cell proliferation since this molecule has a similar chemical structure with ginsenoside Rg1 and Rh1. However, the effect of ginsenoside Rf on cancer cells remains to be elucidated.

Osteosarcoma is the eighth most common form of childhood cancer, comprising $2.4 \%$ of all malignancies in pediatric 
patients and $20 \%$ of all primary bone cancers. The 5-year survival rate of osteosarcoma patients is only $50-70 \%$ due to the lack of effective treatment options (11). Concerning the clinical treatment of cancer, cell cycle control, a major regulatory mechanism of cell proliferation, is one of the most effective methods for cancer treatment through inhibition of cancer cell growth (12). Thus, there are many cytotoxic agents and/or DNA damaging agents that arrest the cell cycle at the G0/G1, S or G2/M phase consequently inducing apoptosis of cancer cells (13). The cyclin/Cdk families have been found to play an important role in $\mathrm{G} 2$ to $\mathrm{M}$ phase progression, particularly at the end of the G2 phase when a threshold level of the active cyclin B1/CDK1 complex, also known as maturation promoting factor (MPF), has been reached, followed by induction of cell apoptosis (14). Apoptosis is the result of a highly complicated cascade of cellular events that result in cell rounding and shrinkage, chromatin condensation, DNA fragmentation, shedding of smaller fragments from cells, and detachment from the plate. In particular, the mitochondria play a central role in the occurrence of apoptosis resulting from many chemotherapeutic agents (15). It is also well known that caspases, such as caspase- $3,-8$ and -9 , which are highly conserved cysteine-dependent aspartate-specific proteases and the key components of the apoptotic machinery, play the central role in apoptotic progression (16).

The present study was designed to clarify the antitumor activity of ginsenoside $\mathrm{Rf}$ in a panel of established cancer cell lines by MTT and colony forming assays. Furthermore, we investigated cell cycle arrest and mitochondrial-associated apoptotic events in the osteosarcoma MG-63 cell line, which is the most sensitive to ginsenoside $\mathrm{Rf}$, in order to elucidate the mechanism of ginsenoside Rf-induced apoptosis in human osteosarcoma.

\section{Materials and methods}

Drugs and materials. Ginsenoside Rf was purchased from the National Institutes for Food and Drug Control (NIFDC) (CAS no. 111719) at a purity of 99.69\%. Dulbecco's modified Eagle's medium (DMEM), fetal calf serum (FCS), trypan blue, penicillin $\mathrm{G}$ and streptomycin were obtained from Gibco-BRL (Gaithersburg, MD, USA). 3-(4,5-Dimethylthiazol-2-yl)-2,5diphenyltetrazolium bromide (MTT), dimethyl sulfoxide (DMSO), ribonuclease (RNase), propidium iodide (PI) and 5-bromo-4-chloro-3-indolyl-phosphate/nitro blue tetrazolium (BCIP/NBT) were purchased from Sigma Chemical (St. Louis, MO, USA). The Apoptosis Detection Kit I was a product of BD Pharmingen. Hoechst 33258, JC-1 and caspase assay kits were purchased from Beyotime (Jiangsu, China). Human reactive monoclonal antibodies against cyclin B1, Cdk1, Bcl-2, Bax, cytochrome $c$ and $\beta$-actin were purchased from Santa Cruz Biotechnology, Inc. (Santa Cruz, CA, USA).

Cell lines and cell culture. Human osteosarcoma cell lines, MG-63 (wild-type), OS732 (wild-type), U-2OS (wild-type), HOS (wild-type) and SAOS-2 (wild-type), were purchased from the Institute of Biochemistry and Cell Biology, The Chinese Academy of Sciences (Shanghai, China). Cells were cultured in DMEM supplemented with $10 \%$ heat-inactivated FBS, $100 \mathrm{U} / \mathrm{ml}$ penicillin and $100 \mu \mathrm{g} / \mathrm{ml}$ streptomycin. They were all placed in a humidified atmosphere containing $5 \%$ $\mathrm{CO}_{2}$ at $37^{\circ} \mathrm{C}$.

Analysis of cell proliferation. The cytotoxicity assay was analyzed with the MTT assay and the trypan blue dye assay. Briefly, MG-63, OS732, U-2OS, HOS and SAOS-2 cells were seeded in 96-well plates at a density of $1 \times 10^{4}$ cells/well with $100 \mu \mathrm{l}$ of the cell culture medium. After $12 \mathrm{~h}$ of incubation, the cells were treated with ginsenoside Rf from 0 to $30 \mu \mathrm{M}$ in medium for $24 \mathrm{~h}$. MTT solution $(5 \mathrm{mg} / \mathrm{ml})$ was then added to each well and incubated at $37^{\circ} \mathrm{C}$ for $4 \mathrm{~h}$. The supernatants were then removed and replaced by $100 \mu \mathrm{l}$ DMSO. The optical density (OD) was measured at a wavelength of $570 \mathrm{~nm}$ with an enzyme immunoassay analyzer (Bio-Rad, USA). The cytotoxicity of ginsenoside $\mathrm{Rf}$ was expressed as $\mathrm{IC}_{50}$ (concentration of $50 \%$ cytotoxicity, which was extrapolated from linear regression analysis of the experimental data).

Colony forming assay. MG-63, OS732, U-2OS, HOS and SAOS-2 cells were seeded at $3 \times 10^{2} /$ well into 24 -well culture plates, respectively, and incubated overnight prior to treatment. Culture medium containing increasing drug concentrations was added to the cells, and incubation was carried out for 7 days in the presence of the drug. After the wells were washed with phosphate-buffered saline (PBS), the colonies were fixed with methanol, stained with crystal violet and counted (>50 cells). The antiproliferative activity was expressed as $\mathrm{EC}_{50}$ (50\% inhibiting concentration).

Analysis of cell cycle distribution by flow cytometry. Cells $\left(2 \times 10^{5}\right)$ were seeded in a 6-well culture plate. After a 12-h incubation, cells were treated with $0,2.75,5.5$ and $11 \mu \mathrm{M}$ of ginsenoside $\mathrm{Rf}$ for $0,12,24,36$ and $48 \mathrm{~h}$, respectively. Both floating and adherent cells were harvested, combined and processed. Cells were collected by centrifugation, fixed with ice-cold $70 \%$ ethanol, washed with PBS, and resuspended in $0.5 \mathrm{ml}$ of PBS containing PI $(500 \mu \mathrm{g} / \mathrm{ml})$ and RNase A $(1 \mathrm{mg} /$ $\mathrm{ml})$. After a final incubation at $37^{\circ} \mathrm{C}$ for $30 \mathrm{~min}$, cells were analyzed using a FACScan flow cytometer (Becton-Dickinson). The percentages of cells in G0/G1 phase, S phase, G2/M and sub-G1 phase were analyzed using standard ModiFit and CellQuest software programs.

Detection of apoptotic incidence by flow cytometry. Apoptotic incidence was measured with the Annexin V-FITC Apoptosis Detection Kit I according to the manufacturer's instructions. Briefly, cells were treated with $0,2.75,5.5$ and $11 \mu \mathrm{M}$ of ginsenoside Rf for $24 \mathrm{~h}$, respectively. The cells were then washed twice with cold PBS, and resuspended in $500 \mu \mathrm{l}$ of binding buffer at a concentration of $1 \times 10^{6}$ cells $/ \mathrm{ml}$. Annexin V-FITC solution $(5 \mu \mathrm{l})$ and $5 \mu \mathrm{l}$ of PI $(1 \mathrm{mg} / \mathrm{ml})$ were added to the cells at $37^{\circ} \mathrm{C}$ for $30 \mathrm{~min}$. The cells were analyzed by flow cytometry within $1 \mathrm{~h}$. Apoptotic cells were counted, and data are expressed as a percentage of the total cell count.

Assay of mitochondrial membrane potential changes in situ. JC-1 probe was employed to measure mitochondrial depolarization in MG-63 cells. Following treatment with $0,2.75,5.5$ and $11 \mu \mathrm{M}$ of ginsenoside $\mathrm{Rf}$ for $24 \mathrm{~h}$, the MG-63 cells were incubated at $37^{\circ} \mathrm{C}$ for $20 \mathrm{~min}$ with $5 \mathrm{mg} / \mathrm{l} \mathrm{JC}-1$, then washed 
twice with PBS and placed in $2 \mathrm{ml}$ culture medium. Green fluorescence (JC-1 as a monomer at low membrane potentials) and red fluorescence (JC-1 as 'J-aggregates' at higher membrane potentials) were monitored under a fluorescence microscope. Mitochondrial depolarization was indicated by a decrease in the red/green fluorescence intensity ratio.

Hoechst 33258 staining of MG-63 cells. Cells were incubated with $0,2.75,5.5$ and $11 \mu \mathrm{M}$ of ginsenoside $\mathrm{Rf}$ for $24 \mathrm{~h}$, harvested, fixed with $4 \%$ paraformaldehyde for $30 \mathrm{~min}$ at $25^{\circ} \mathrm{C}$, washed 3 times with ice-cold PBS, and exposed to $10 \mathrm{mg} / \mathrm{l}$ Hoechst 33258 in the dark at room temperature for $10 \mathrm{~min}$. Finally, the stained nuclei were observed under a fluorescence microscope (Olympus x50) with excitation at $350 \mathrm{~nm}$ and emission at $460 \mathrm{~nm}$.

Assays of caspase activity. Caspase activity was determined using caspase assay kits. According to the manufacturer's protocol, cells were placed in $10-\mathrm{cm}$ dishes at $1 \times 10^{6} / \mathrm{dish}$. After a 12-h incubation, cells were treated with $0,2.75,5.5$ and $11 \mu \mathrm{M}$ of ginsenoside Rf for $24 \mathrm{~h}$. After the different treatments, both floating and adherent cells were collected and washed 3 times by PBS and were lysed with lysis buffer $\left(100 \mu \mathrm{l} / 2 \times 10^{6}\right.$ cells) for $15 \mathrm{~min}$ on ice. Cell lysates were centrifuged at $16,000 \mathrm{x} \mathrm{g}$ for $10 \mathrm{~min}$ at $4^{\circ} \mathrm{C}$. Then, clear lysates containing $50 \mu \mathrm{g}$ of protein were incubated with $100 \mu \mathrm{M}$ of enzyme-specific colorigenic substrates at $37^{\circ} \mathrm{C}$ for $1 \mathrm{~h}$. The activities of caspase-3, -8 and -9 were described as the cleavage of the colorimetric substrate by measuring the absorbance at $405 \mathrm{~nm}$ with a microplate spectrophotometer (BioTek), respectively.

Western blotting. Cells were placed in $10-\mathrm{cm}$ dishes at $1 \times 10^{6} / \mathrm{dish}$. After a 12 -h incubation, cells were treated with $0,2.75,5.5$ and $11 \mu \mathrm{M}$ of ginsenoside $\mathrm{Rf}$ for $24 \mathrm{~h}$. Total protein was extracted using a Western \& IP cell lysis kit after treatment, or cytoplasmic protein was extracted using a Mitochondrial Isolation kit (Applygen). For total cell protein extracts, the treated cells were washed in PBS, suspended in lysis buffer and placed in ice for $30 \mathrm{~min}$. After centrifugation for $15 \mathrm{~min}$ at $4^{\circ} \mathrm{C}$, the supernatant was collected. Total proteins were prepared by standard procedures and quantified by the BCA method (Pierce Biotechnology, Inc., Rockford, IL, USA).

The western blot assay was performed as described previously. Briefly, whole cell extracts were separated by $8 \%$ sodium dodecyl sulfate-polyacrylamide gel electrophoresis (SDS-PAGE) and electrotransferred to polyvinylidene difluoride (PVDF) membranes (Bio-Rad, Hercules, CA, USA). After the PVDF membranes were incubated with $10 \mathrm{mM}$ TBS with $1.0 \%$ Tween-20 and $10 \%$ dehydrated skim milk to block nonspecific protein binding, the membranes were incubated with the appropriate primary antibodies in blocking buffer overnight at $4^{\circ} \mathrm{C}$. The membranes were then washed with TBST and incubated with alkaline phosphatase-linked secondary antibodies (Pierce Biotechnology, Inc.). After being washed with TBST, immunoreactive bands were visualized using NBT/BCIP as substrate. $\beta$-actin was used as a loading control.

RT-PCR analysis of cyclin B1 and Cdk1 mRNA. Total RNA of the ginsenoside Rf-treated cells extracted using $1 \mathrm{ml}$
Table I. Growth inhibitory effect of ginsenoside Rf on 5 osteosarcoma cell lines.

\begin{tabular}{lrrrcc}
\hline & OS732 & MG-63 & U-2OS & HOS & SAOS-2 \\
\hline $\mathrm{IC}_{50}(\mu \mathrm{M})$ & 20.43 & 11.36 & 25.02 & $>30$ & $>30$ \\
$\mathrm{EC}_{50}(\mu \mathrm{M})$ & 6.84 & 0.93 & 8.58 & $/$ & $/$ \\
$\mathrm{TI}$ & 2.98 & 12.21 & 2.91 & $/$ & $/$ \\
\hline
\end{tabular}

Each value represents the mean \pm standard deviation $(\mathrm{SD})$ of 3 independent experiments. Therapeutic index (TI) is equal to the ratio of $\mathrm{IC}_{50}$ vs. $\mathrm{EC}_{50}$. The $\%$ ' indicates no sense.

TRIzol reagent (Invitrogen Life Technologies) was dissolved in $0.1 \%$ diethylpyrocarbonate (DEPC) water and quantified by spectrophotometry at $260 \mathrm{~nm}$ (absorbance). cDNA was synthesized from $1 \mu \mathrm{g}$ total RNA through reverse transcription using a Takara RNA PCR kit ver. 2.1 (Takara Bio, Inc.) according to the manufacturer's protocol. Primers were obtained from Shanghai Sangon Biotechnology Co. Ltd. (Shanghai, China), and the sequences for the primers are as follows: 5'-CTTATACTAAGCACCAAATC-3' (sense) and 5'-CTTGGCTAAATCTTGAACT-3' (antisense) for cyclin B1; 5'-CTTATGCAGGATTCCAGGTT-3' (sense) and 5'-GGTGCCTATACTCCAAATGTC-3' (antisense) for Cdk1; and 5'-GGTCGGAGTCAACGGATTTG-3' (sense) and 5'-ATGAGCCCCAGCCTTCTCCAT-3' (antisense) for GAPDH. Quantitative real-time PCR was performed using $1 \mu \mathrm{g}$ of cDNA and SYBR-Green (Bio-Bad) in 36-well plates in a LightCycler Rapid Thermal Cycler system (Roche Diagnostics) according to the manufacturer's instructions. PCR products were subjected to melting curve analysis, and the data were analyzed by the $2^{-\Delta \Delta C t}$ calculation method and standardized to GAPDH. PCR analysis was performed in triplicate for each sample.

Statistical analysis. All data are expressed as the mean \pm standard deviation (SD). Statistical analysis was performed using the SPSS 17.0 statistical software program to evaluate the significance of differences between groups considered as $\mathrm{p}<0.05 ; \mathrm{p}<0.01 ; \mathrm{p}<0.001$. All data points represent the mean of triplicate values.

\section{Results}

Effect of ginsenoside Rf on cell proliferation in human osteosarcoma cell lines. To clearly identify the antiproliferative effect of ginsenoside Rf on human osteosarcoma cell lines, we initially treated 5 human osteosarcoma cell lines (MG-63, OS732, U-2OS, HOS and SAOS-2) with ginsenoside Rf at different concentrations for 12, 24, 36 and $48 \mathrm{~h}$, respectively. Cell proliferation and cell viability were estimated by the MTT assay and trypan blue staining. As shown in Fig. 1, the cytotoxicity of ginsenoside $\mathrm{Rf}$ to the human osteosarcoma cell lines was dose-dependent, and each cell line exhibited a different sensitivity to ginsenoside Rf. Notably, the MG-63 cells were the most sensitive to ginsenoside $\mathrm{Rf}$. The $\mathrm{IC}_{50}$ values for the cytotoxic effect are showed in Table $\mathrm{I}$. The $\mathrm{IC}_{50}$ 

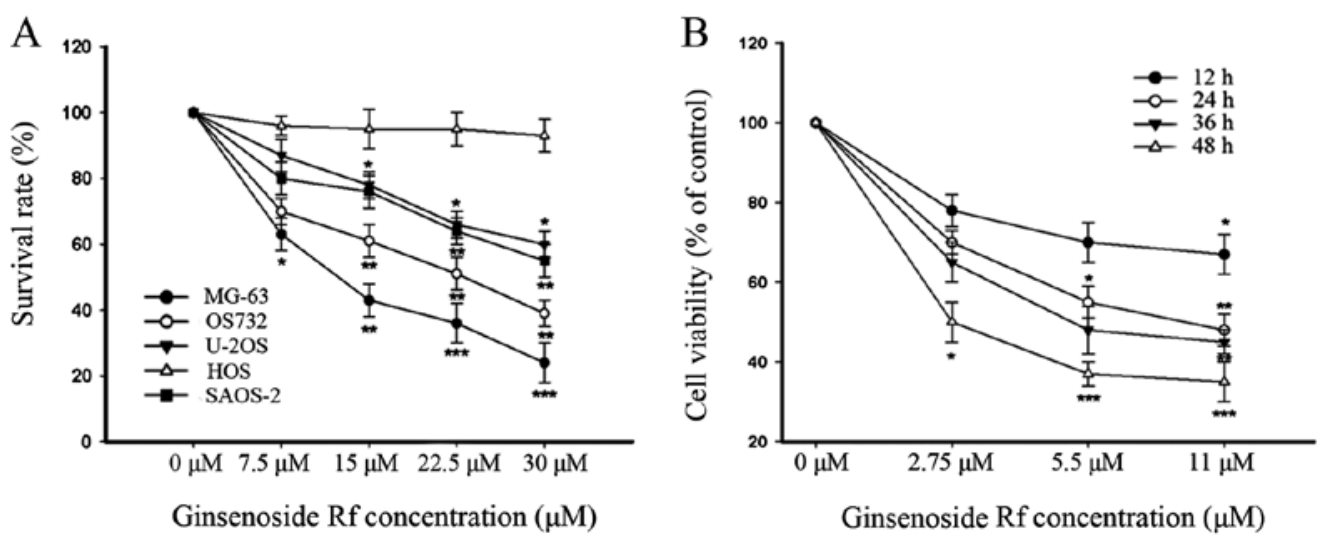

Figure 1. Analysis of cell viability. (A) Effect of ginsenoside Rf on the viability of MG-63, OS732, U-2OS, HOS and SAOS-2 cells. Cells were treated with ginsenoside Rf at different concentrations for $24 \mathrm{~h}$. (B) Dose- and time-effect of ginsenoside Rf on the viability of MG-63 cells. Cells were treated with ginsenoside $\mathrm{Rf}$ at different concentrations for 12,24,36 and $48 \mathrm{~h}$. Cell viability was then determined and expressed as means \pm SD of 3 separate experiments. Significant differences from control $(0 \mu \mathrm{M})$ are indicated by ${ }^{*} \mathrm{p}<0.05 ;{ }^{* *} \mathrm{p}<0.01 ;{ }^{* * *} \mathrm{p}<0.001$.

value for the MG-63 cells treated with ginsenoside Rf was $11 \mu \mathrm{M}$ at $24 \mathrm{~h}$. This dose- and time-point were referred to in the following experiments. Doses of $0,2.75$ and $5.5 \mu \mathrm{M}$ were used in the following assays. Our findings demonstrated that ginsenoside $\mathrm{Rf}$ inhibited cellular proliferation in a time- and dose-dependent manner.

Effect of ginsenoside $R f$ on the colony forming ability of human osteosarcoma cell lines. The $\mathrm{EC}_{50}$ values for colony formation are showed in Table I. The therapeutic indices (the ratio of $\mathrm{IC}_{50} \mathrm{vs} . \mathrm{EC}_{50}$ ) for the 5 human osteosarcoma cell lines were in the following order: MG-63, OS732, U-2OS, HOS and SAOS-2 successively. This suggested that MG- 63 cells, which were used in the following experiments, were most sensitive to ginsenoside $\mathrm{Rf}$ with a therapeutic index $\left(\mathrm{IC}_{50} \mathrm{vs} . \mathrm{EC}_{50}\right)$ equal to 12.21 .

Ginsenoside Rf induces G2/M phase arrest and apoptosis in MG-63 cells. To determine whether changes in cell cycle distribution were involved in the decrease in cell viability, we investigated the effect of ginsenoside Rf on cell cycle distribution by flow cytometric analysis. The exposure of cells to 0 , $2.75,5.5$ and $11 \mu \mathrm{M}$ ginsenoside $\mathrm{Rf}$ for $24 \mathrm{~h}$ resulted in the accumulation of the proportion of cells in the $\mathrm{G} 2 / \mathrm{M}$ phase from $13.26 \pm 2.6 \%$ to $15.63 \pm 2.9,19.22 \pm 4.2$ and $49.55 \pm 4.7 \%$, respectively (Fig. 2A). Ginsenoside Rf resulted in a time-dependent G2/M phase cell cycle arrest, and G2/M phase accumulation peaked following treatment with $11 \mu \mathrm{M}$ ginsenoside $\mathrm{Rf}$ at $24 \mathrm{~h}$ (Fig. 2B). These results suggest that ginsenoside $\mathrm{Rf}$ inhibits the cellular proliferation via $\mathrm{G} 2 / \mathrm{M}$ phase cell cycle arrest in a time- and dose-dependent manner.

The rate of cell apoptosis was detected by flow cytometry by double labeling with Annexin V and PI. Representative graphs obtained by flow cytometric analysis of cells treated with ginsenoside $\mathrm{Rf}$ at different concentrations for $24 \mathrm{~h}$ after double staining with Annexin V-FITC and PI are shown in Fig. 2C. The apoptotic rate in the control cells was $2.1 \pm 0.7 \%$. There was a dose-dependent increase in the apoptotic rate of MG-63 cells exposed to ginsenoside Rf. The apoptotic rates in the MG-63 cells were increased to $4.6 \pm 1.6,14.9 \pm 2.9$ and
$29.6 \pm 3.8 \%$ following treatment with ginsenoside $\mathrm{Rf}$ at $2.75,5.5$ and $11 \mu \mathrm{M}$ for $24 \mathrm{~h}$, respectively (Fig. 1D). Using Hoechst 33258 staining, we further confirmed that ginsenoside $\mathrm{Rf}$ induced MG-63 cell death through the apoptosis pathway (Fig. 2E). MG-63 cells treated with $11 \mu \mathrm{M}$ ginsenoside Rf for $24 \mathrm{~h}$ exhibited an increase in chromatin condensation and nuclear fragmentation. The results indicate that cell death occurred through apoptosis.

Effect of ginsenoside $R f$ on mitochondrial membrane potential in MG-63 cells. In order to investigate whether the mitochondrial pathway is involved in the apoptosis induced by ginsenoside Rf, we analyzed MG-63 cells treated with ginsenoside $\mathrm{Rf}$ at different concentrations for $24 \mathrm{~h}$ using the membrane potential sensing dye JC-1. The control cells stained with JC-1 emitted mitochondrial red fluorescence with slight green fluorescence, indicative of a normal polarized state. The JC-1 aggregates were dispersed to the monomeric form (green fluorescence) in the cells exposed to $11 \mu \mathrm{M}$ ginsenoside $\mathrm{Rf}$ for $24 \mathrm{~h}$ (Fig. 3A). The results showed that ginsenoside $\mathrm{Rf}$ reduced the ratio of red (JC-1 aggregates) fluorescence to green (JC-1 monomers) fluorescence, indicating that ginsenoside $\mathrm{Rf}$ caused the dissipation of mitochondrial membrane potential (Fig. 3B).

Effect of ginsenoside Rf on Cdkl and cyclin B1 expression. In an attempt to investigate the molecular events involved in the activity of ginsenoside Rf activity on the cell cycle, we assessed the effect of ginsenoside Rf on the expression of Cdk1 and cyclin B1 in MG-63 cells. The protein levels of Cdk1 and cyclin B1 were detected by western blotting after MG- 63 cells were treated with $0,2.75,5.5$ and $11 \mu \mathrm{M}$ ginsenoside $\mathrm{Rf}$ for $24 \mathrm{~h}$. As shown in Fig. 4A and B, the level of cyclin B1 protein in the cells treated with $11 \mu \mathrm{M}$ ginsenoside Rf increased almost $1 / 5$-fold over the control group at $24 \mathrm{~h}$. As expected, we also found that the expression of Cdk1 protein was attenuated in the cells treated with $11 \mu \mathrm{M}$ ginsenoside Rf, which was only $\sim 1 / 4$ times more than the NC group. The changes in Cdk1 and cyclin B1 mRNA expression, as measured by real-time PCR, were almost parallel with that of the corresponding protein (Fig. 4C). Our results suggest that downregulation of 
A

C

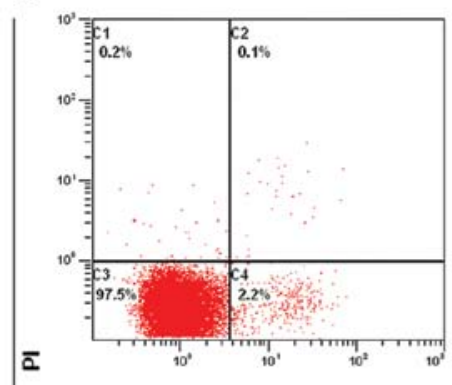

$0 \mu \mathrm{M}$

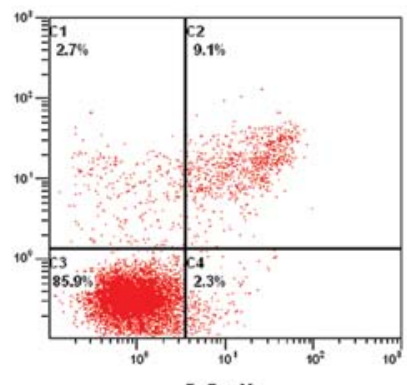

$5.5 \mu \mathrm{M}$

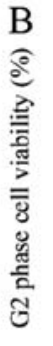

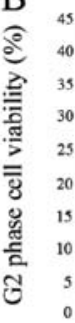

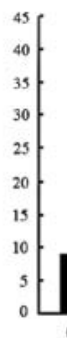

L.

Time (

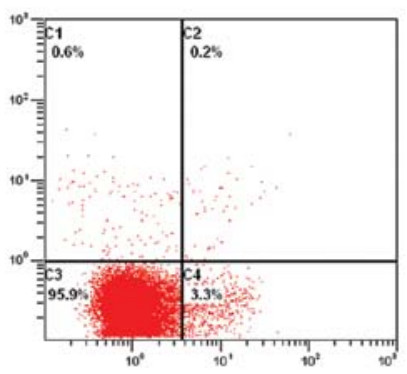

2. $75 \mu \mathrm{N}$

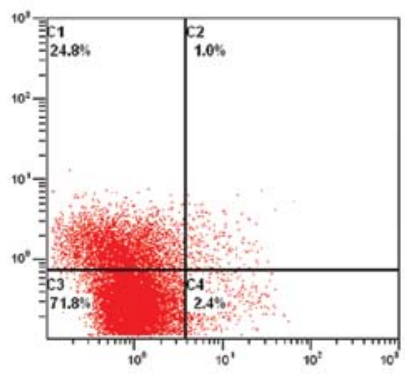

$11 \mu \mathrm{M}$

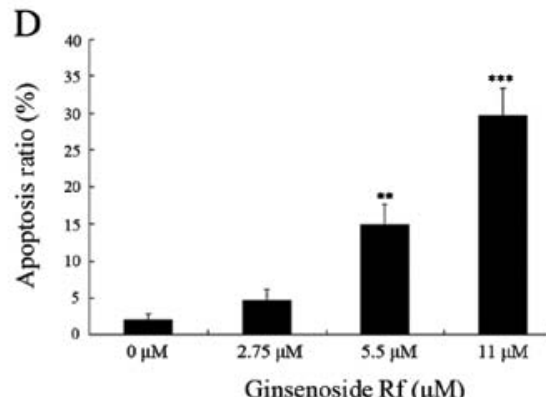

E

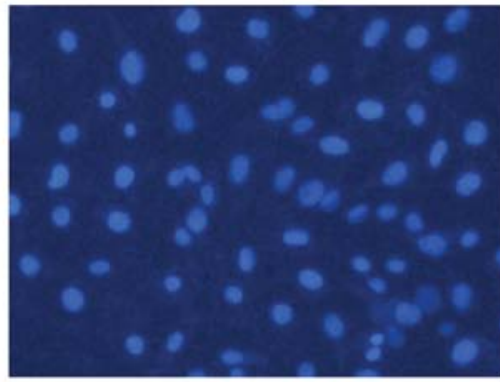

Ginsenoside Rf $(0 \mu \mathrm{M})$

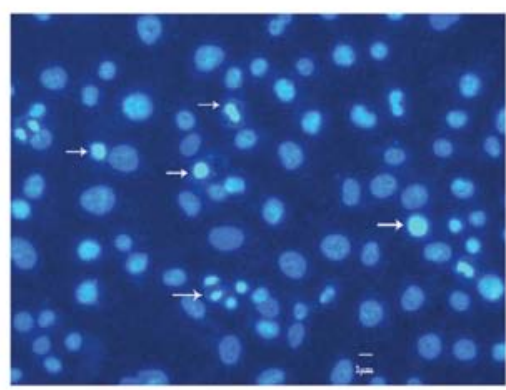

Ginsenoside Rf $(11 \mu \mathrm{M})$

Figure 2. Ginsenoside Rf induces G2/M phase cell cycle arrest and apoptosis in MG-63 cells. (A) Dose-dependent effect of ginsenoside Rf on the cell cycle distribution of MG-63 cells. The histogram shows the percentage of cells/channel (vertical axis) vs. DNA content (horizontal axis). The values indicate the percentage of cells in the indicated phases of the cell cycle. (B) Time-dependent effect of ginsenoside Rf on the population of G2 phase cells after MG-63 cells were treated with ginsenoside $\operatorname{Rf}(11 \mu \mathrm{M})$. The percentage of G2 phase cells showed a time-dependent response to ginsenoside Rf. G2 phase accumulation peaked at $24 \mathrm{~h}$ of treatment. (C) Representative graphs obtained by flow cytometric analysis after double-staining with Annexin V-FITC and PI. (D) The percentage of apoptotic MG-63 cells following treatment with ginsenoside $\mathrm{Rf}$ at $0,2.75,5.5$ and $11 \mu \mathrm{M}$ for $24 \mathrm{~h}$. Compared with the control cells $(0 \mu \mathrm{M}, 2.1 \pm 0.7 \%)$, the percentage of apoptotic MG-63 cells was obviously increased to 4.6 $\pm 1.6,14.9 \pm 2.9$ and $29.6 \pm 3.8 \%$ after treatment for $24 \mathrm{~h}$, respectively. (E) Hoechst 33258 staining of MG-63 cells. Apoptotic nuclei exhibited condensed or fragmented DNA brightly stained by Hoechst 33258 (arrows, 24 h). Original magnification, $\mathrm{x} 200$. Significant differences from the control are indicated by ${ }^{*} \mathrm{p}<0.05 ;{ }^{* *} \mathrm{p}<0.01 ;{ }^{* * *} \mathrm{p}<0.001$.

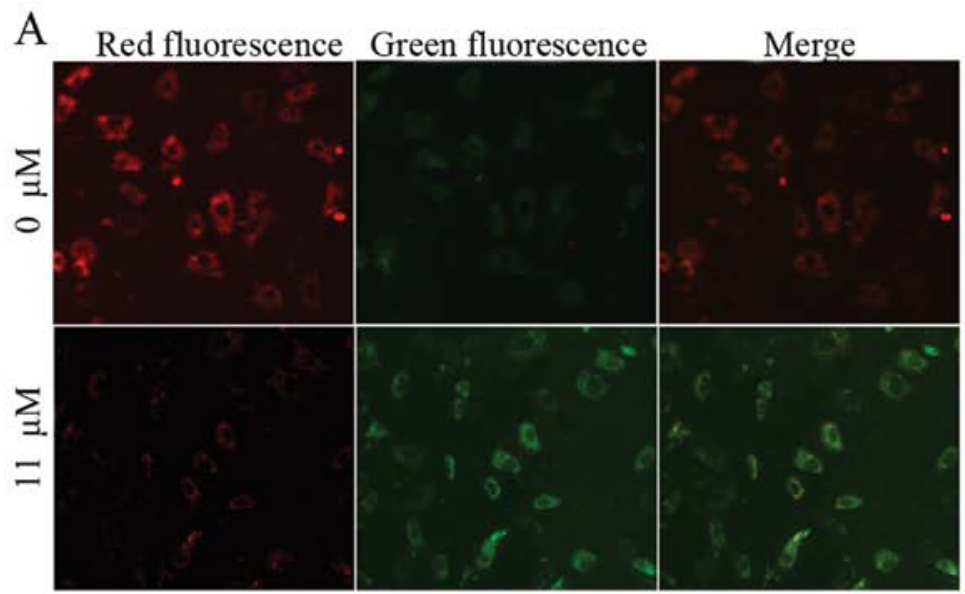

B

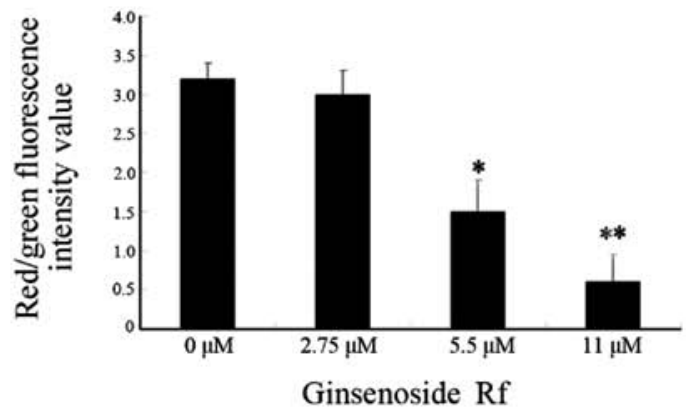

Figure 3. Ginsenoside Rf decreases the mitochondrial membrane potential in MG-63 cells. (A) Representative images of JC-1 staining in the different groups The two images in each row were captured under the same field and were then merged. (B) Quantitative analysis of the shift of mitochondrial red fluorescence to green fluorescence in the groups. Red/green fluorescence intensity value was calculated. All values are showed as means \pm SD from 12 independent images captured in each group. ${ }^{*} \mathrm{p}<0.05$ vs. control; ${ }^{* *} \mathrm{p}<0.01$ vs. control. 
A
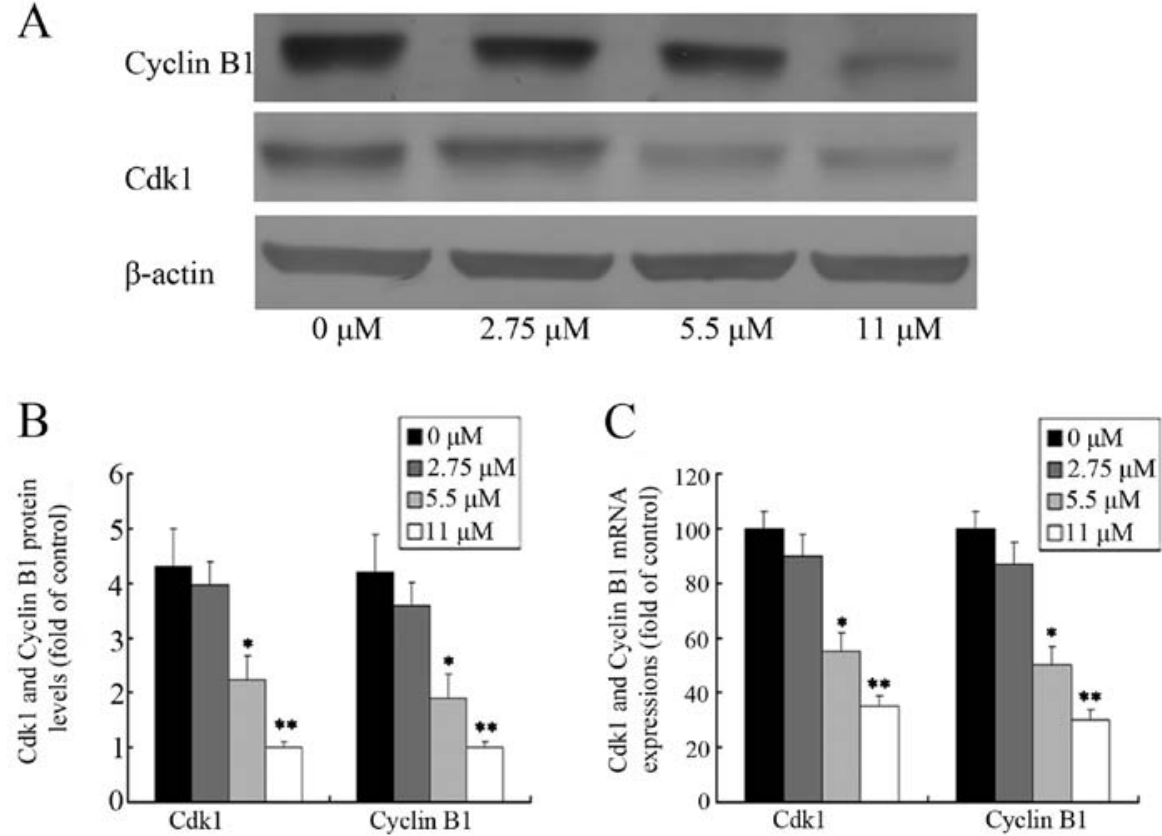

Figure 4. Expression of Cdk1 and cyclin B1 is decreased in MG-63 cells following treatment with ginsenoside Rf. (A) Representative western blotting for Cdk1 and cyclin B1 in MG-63 cells treated with 0, 2.75, 5.5 and $11 \mu \mathrm{M}$ ginsenoside Rf for $24 \mathrm{~h}$. (B) Quantitative analysis of Cdk1 and cyclin B1 protein levels. The values from treated cells were normalized to values in the control cells. (C) mRNA expression of Cdk1 and cyclin B1 by RT-PCR in the different groups. The values from treated cells were normalized to GAPDH and then expressed as a ratio of normalized values to mRNA in the control cells $(0 \mu \mathrm{M})$. Significant differences from the control cells $(0 \mu \mathrm{M})$ are indicated by ${ }^{*} \mathrm{p}<0.05 ;{ }^{* *} \mathrm{p}<0.01 ;{ }^{* * *} \mathrm{p}<0.001$.

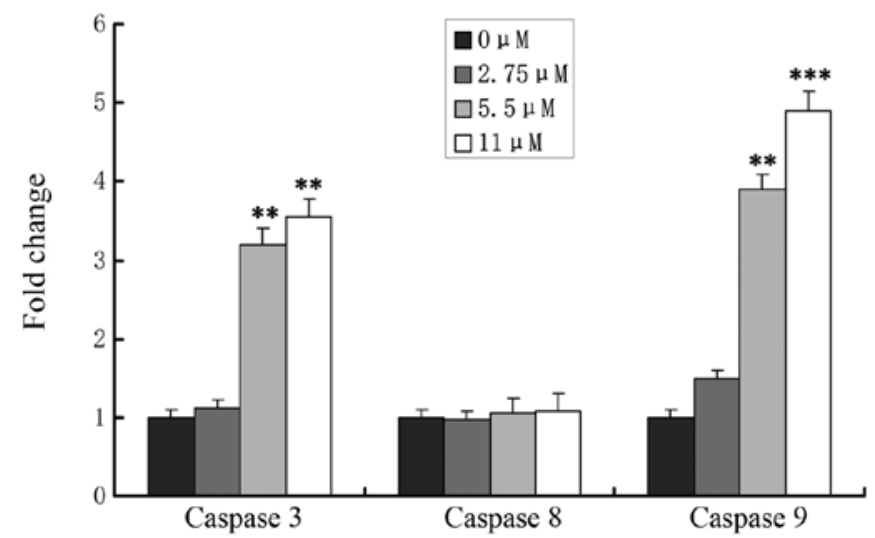

Figure 5. Effects of ginsenoside Rf on caspase- $3,-8$ and -9 activation. After treatment with $0,2.75,5.5$ and $11 \mu \mathrm{M}$ of ginsenoside $\mathrm{Rf}$ for $24 \mathrm{~h}$, significant increases in caspase- 3 and -9 activity were noted but not for caspase-8. Significant differences from control cells are indicated by ${ }^{* *} \mathrm{p}<0.01$; **** $\mathrm{p}<0.001$.

the expression of $\mathrm{G} 2$ phase-regulating proteins may contribute to the ginsenoside Rf-mediated cell cycle arrest in MG-63 cells.

Effects of ginsenoside Rf on caspase activation. The caspase cascade reaction is one of the most important events in the process of apoptosis through the mitochondrial pathway. To determine whether release of caspase-3, -8 and -9 is involved in ginsenoside Rf-induced apoptosis, MG-63 cells were treated with $0,2.75,5.5$ and $11 \mu \mathrm{M}$ ginsenoside $\mathrm{Rf}$, and caspase-8, -9 and caspase- 3 activation was determined at $24 \mathrm{~h}$ (Fig. 5). We assessed the caspase-3 and -9 levels in cell lysates and found that caspase-3 and -9 levels increased significantly in the MG-63 cells in a dose-dependent manner, which indicated that mitochondrial pathways were involved in ginsenoside Rf-induced apoptosis. Simultaneously, we found that activity of caspase- 8 was not changed in the cells treated with ginsenoside Rf.

Effect of ginsenoside $R f$ on the expression of apoptosisrelated proteins. The loss of mitochondrial transmembrane potential and the release of cytochrome $c$ from mitochondria to the cytosol are pivotal events in the mitochondrial apoptosis pathway, which is regulated by Bcl-2 family members (such as Bcl-2 and Bax) during this process $(17,18)$. To explore the possible role of Bcl-2 family members and cytochrome $c$ in ginsenoside Rf-induced apoptosis, we investigated the effect of ginsenoside $\mathrm{Rf}$ at $0,2.75,5.5$ and $11 \mu \mathrm{M}$ for $24 \mathrm{~h}$ on the expression of proteins including Bcl-2, Bax and the release of cytochrome $c$ by western blotting. As shown in Fig. 6, treatment of MG-63 cells with ginsenoside Rf caused a marked increase in Bax proteins and the release of cytochrome $c$, and a decrease in Bcl-2 protein, when compared to these levels in the control.

\section{Discussion}

The aim of the present study was to clarify whether ginsenoside Rf has a cytotoxic effect on human osteosarcoma cells and to explore the molecular mechanisms involved during this process. The main findings of our study are as follows. First, we demonstrated that the cytotoxicity of ginsenoside Rf to human osteosarcoma cell lines was dose-dependent and that the MG-63 cells were the most sensitive to ginsenoside Rf. Second, we showed that ginsenoside Rf induced G2/M phase 


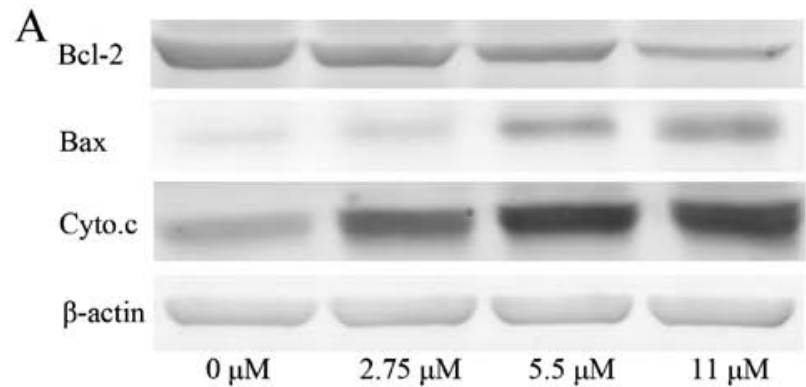

B

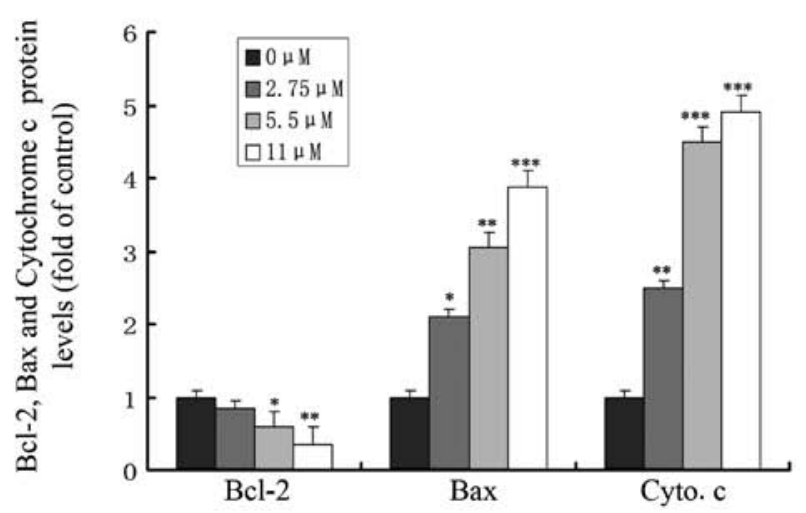

Figure 6. Activation of Bcl-2, Bax and accumulation of cytosolic cytochrome $c$ during ginsenoside Rf-induced apoptosis. (A) Western blot analysis of protein extracts obtained from MG-63 cells treated with $0,2.75,5.5$ and $11 \mu \mathrm{M}$ ginsenoside Rf, respectively. Total protein extracts or cytosolic fraction of cells were prepared after treatment for $24 \mathrm{~h}$, and analyzed with antibodies to Bcl-2, Bax and cytochrome $c$. $\beta$-actin was used as a loading control. (B) Quantitative analysis of Bcl-2, Bax and cytochrome $c$ protein levels. The values from treated cells were normalized to values in the control cells. Significant differences from the control cells are indicated by ${ }^{*} \mathrm{p}<0.05 ;{ }^{* *} \mathrm{p}<0.01 ;{ }^{* * *} \mathrm{p}<0.001$.

cell cycle arrest and cell death through the apoptosis pathway. Furthermore, we found that the mitochondrial pathway participated in the process of apoptosis induced by ginsenoside Rf.

Conventional chemotherapeutic agents kill cancer cells as well as normal cells, limiting their therapeutic use in the clinic. Previous studies have shown that natural bioactive reagents as an approach to effective cancer prevention can selectively kill cancer cells, based on their favorable safety and efficacy profiles (19). Ginseng, a widely used traditional Chinese medicine, has been reported to exhibit various biological effects including antitumor activity (1). The antitumor efficacy of ginseng is attributed mainly to the presence of saponins, known as ginsenosides. Previous studies have demonstrated that several ginsenosides such as the ginsenoside $\mathrm{Rg} 3$ and $\mathrm{Rh} 2$ lead to the accumulation of cells in the G2/M phase and cell apoptosis by DNA damaging agents $(20,21)$. However, whether ginsenoside Rf, which is unique to Asian ginseng, influences human osteosarcoma cell viability and regulates cell apoptosis has not been reported. Here, we first investigated the cytotoxicity of ginsenoside Rf to MG-63, OS732, U-2OS, HOS and SAOS-2 cells. According to our results, ginsenoside Rf inhibited cell proliferation in the human osteosarcoma cell lines in a dose-dependent manner. MG-63 cells were the most sensitive to ginsenoside Rf. The value of $\mathrm{IC}_{50}$ in the MG-63 cells following treatment with ginsenoside $\mathrm{Rf}$ was found to be $11 \mu \mathrm{M}$ at $24 \mathrm{~h}$. Therefore, we chose this dose- and time-point as a reference for MG-63 cells in other experiments.

The cell cycle is mediated by the activation of a highly conserved protein kinase family, the cyclin-dependent kinases (Cdks) (22). Cyclins can activate Cdks by forming complexes with Cdks, and these cyclin/Cdk complexes are cell cycle regulators. Among them, the cyclin B1/Cdk1 complex, in which B-type cyclins associate with Cdk1, is the primary regulator of transition from the $\mathrm{G} 2$ to $\mathrm{M}$ phase. This complex was originally discovered and defined as the maturationpromoting factor or M phase-promoting factor (MPF). The cell cycle arrests at the $\mathrm{G} 2$ phase and mitosis cannot occur without activation of the cyclin B1/Cdk1 complex. In the present study, flow cytometric analysis showed that ginsen- oside Rf caused G2/M phase cell cycle arrest, and G2/M phase accumulation peaked at $24 \mathrm{~h}$ of treatment. Subsequently, the apoptotic sub-G1 phase increased obviously after $>24 \mathrm{~h}$ of treatment, suggesting the occurrence of sequential events of cell cycle arrest followed by apoptosis; it is known that cell cycle dysregulation is a hallmark of tumor cells. Evidence indicates that $\mathrm{G} 2 / \mathrm{M}$ phase arrest is mediated by a limited supply of Cdk1 and cyclin B1 for Cdk1/cyclin B1 complex formation, resulting in the shortage of the cyclin B1/Cdk1 complex during cell cycle progression (23). In the present study, we found that ginsenoside Rf arrested MG-63 cells in the $\mathrm{G} 2 / \mathrm{M}$ phase through a decrease in the cyclin $\mathrm{B} 1 / \mathrm{Cdk} 1$ complex resulting from inhibition of cyclin B1 and Cdk1 expression in a dose-dependent manner at both the transcriptional and protein levels. According to the results mentioned above, ginsenoside $\mathrm{Rf}$ induced $\mathrm{G} 2 / \mathrm{M}$ phase arrest in MG-63 cells by the downregulation of Cdk1 and cyclin B1.

Apoptosis, first recognized by Kerr et al (24), is originally described as a series of morphological events that give rise to controlled cell death and is executed by an active cellular process. This fundamental process is essential for both development and maintenance of tissue homeostasis. There are two principal signaling transduction pathways involved in the process of apoptosis: one is the cell surface death receptor pathway, and the other is the mitochondrial pathway initiated by the upregulation of wild-type p53, followed by suppression of Bcl-2. Moreover, these two pathways can be connected via Bid, one of the Bcl-2 family members (25). In the present study, the inhibition of cell viability was observed in ginsenoside Rf-treated MG-63 cells. After treatment, cells gradually exhibited cell rounding and shrinkage, vacuolization and even detachment from the bottom in a time-dependent manner, which are important characteristics of cell apoptosis. This was further supported by our findings of increased chromatin condensation and nuclear fragmentation over time as observed by Hoechst 33258 staining. Our findings demonstrated that ginsenoside $\mathrm{Rf}$ may serve as a protective agent in cancer treatment as we observed that the apoptosis ratio increased to $29.6 \pm 3.8 \%$ in MG-63 cells following treatment with ginsen- 
oside Rf at $11 \mu \mathrm{M}$ for $24 \mathrm{~h}$ when compared to control cells $(2.1 \pm 0.7 \%)$, which is consistent with previous reports on other ginsenosides (such as ginsenosides Rg1) (26).

The Bcl-2/Bax ratio appears to be a critical determinant of a cell's threshold for undergoing apoptosis through the mitochondrial pathway (27). Our results showed that upregulation of Bax and downregulation of Bcl-2 occurred simultaneously in the human osteosarcoma cell line MG-63 following treatment with ginsenoside Rf, which triggered progression of apoptosis. Therefore, we inferred that the decrease in the $\mathrm{Bcl}-2 / \mathrm{Bax}$ ratio modulated by ginsenoside $\mathrm{Rf}$ induced apoptosis of MG-63 cells. This hypothesis was further supported by nuclear morphological changes using Hoechst 33258 staining. The mitochondrial-mediated apoptosis pathway is triggered by various cellular stress stimuli and is dependent on mitochondrial outer membrane permeabilization, resulting in the loss of mitochondrial transmembrane potential and the release of cytochrome $c$ from the mitochondria to the cytosol (18). Thus, cells use cytosolic cytochrome $c$ as a cue to activate the apoptotic machinery (28). Our results demonstrate that MG-63 cells treated with ginsenoside Rf exhibited a reduction in mitochondrial transmembrane potential. Using western blot analysis, a dose-dependent increase in cytochrome $c$ accumulation was found in the cytosol of ginsenoside Rf-treated MG-63 cells. These results suggest that ginsenoside $\mathrm{Rf}$ stimulates the reduction in mitochondrial transmembrane potential and the release of mitochondrial cytochrome $c$, finally activating the mitochondrial-mediated apoptosis pathway.

In order to clarify the effect of caspases in the apoptotic process, we determined the activation of caspase- $3,-8$ and -9 . Caspases, a family of cysteine proteases, play essential roles in apoptosis, necrosis and inflammation. The activation of caspase-8, which in turn activates downstream caspases, leads to apoptosis through the death receptor pathway, whereas the activation of caspase- 9 , which forms the apoptosome with released cytochrome $c$ and Apaf-1, activates the executioner caspase- 3 and induces apoptosis through the mitochondrial pathway. In the present study, consistent with the study of Rannou et al (29), we also found that ginsenoside Rf had no effect on caspase- 8 activity, suggesting that the death receptormediated apoptotic pathway was not involved in the apoptosis induced by ginsenoside Rf in MG-63 cells. Notably, we found that ginsenoside $\mathrm{Rf}$ enhanced caspase- 3 and -9 activities in a dose-dependent manner, indicating that the mitochondrial death pathway was involved in the process of apoptosis induced by ginsenoside $\mathrm{Rf}$. These results are in concordance with those reported in another study, showing a linkage between activation of caspase- 9 cleavage and activation of procaspase-3 consequently leading to activation of the caspase cascade and apoptosis (30).

In conclusion, our results demonstrated that ginsenoside $\mathrm{Rf}$ inhibits cell proliferation and induces cell cycle arrest at the G2/M phase and apoptosis in human osteosarcoma cells in a dose- and time-dependent manner. The cell cycle arrest induced by ginsenoside $\mathrm{Rf}$ was associated with a reduction in the protein and mRNA levels of Cdk1 and cyclin B1. Furthermore, we found that ginsenoside $\mathrm{Rf}$ decreased the $\mathrm{Bcl}-2 / \mathrm{Bax}$ ratio and the mitochondrial transmembrane potential, resulting in the release of cytochrome $c$ and activation of caspase- 9 and -3 , suggesting that the mitochondrial pathway may be involved in the process of apoptosis induced by ginsenoside Rf in MG-63 cells. In view of the above arguments and the new data presented herein, ginsenoside $\mathrm{Rf}$ can induce G2/M phase arrest and apoptosis in human osteosarcoma MG-63 cells through the mitochondrial pathway. It may be a new effective and promising therapeutic agent for human osteosarcoma, although further research must be carried out to fully investigate these possibilities.

\section{Acknowledgements}

The present study was supported by the National Natural Science Foundation of China (no. 81000779), the Excellent College Teacher Foundation of Shanghai Education Commission, and the Shanghai Jiaotong University SMC Foundation.

\section{References}

1. Nag SA, Qin JJ, Wang W, et al: Ginsenosides as anticancer agents: in vitro and in vivo activities, structure-activity relationships, and molecular mechanisms of action. Front Pharmacol 3: $25,2012$.

2. Fishbein AB, Wang CZ, Li XL, et al: Asian ginseng enhances the anti-proliferative effect of 5-fluorouracil on human colorectal cancer: comparison between white and red ginseng. Arch Pharm Res 32: 505-513, 2009.

3. Kim HS, Lee EH, Ko SR, et al: Effects of ginsenosides Rg3 and $\mathrm{Rh} 2$ on the proliferation of prostate cancer cells. Arch Pharm Res 27: 429-435, 2004.

4. Iishi H, Tatsuta M, Baba M, et al: Inhibition by ginsenoside Rg3 of bombesin-enhanced peritoneal metastasis of intestinal adenocarcinomas induced by azoxymethane in Wistar rats. Clin Exp Metastasis 15: 603-611, 1997.

5. Chen J, Peng H, Ou-Yang X and He X: Research on the antitumor effect of ginsenoside Rg3 in B16 melanoma cells. Melanoma Res 18: 322-329, 2008.

6. Wu N, Wu GC, Hu R, Li M and Feng H: Ginsenoside Rh2 inhibits glioma cell proliferation by targeting microRNA-128. Acta Pharmacol Sin 32: 345-353, 2011.

7. Zhang C, Yu H and Hou J: Effects of 20 (S) -ginsenoside Rh2 and $20(\mathrm{R})$-ginsenoside $\mathrm{Rh} 2$ on proliferation and apoptosis of human lung adenocarcinoma A549 cells. Zhongguo Zhong Yao Za Zhi 36: 1670-1674, 2011 (In Chinese).

8. Qi LW, Wang CZ and Yuan CS: American ginseng: potential structure-function relationship in cancer chemoprevention. Biochem Pharmacol 80: 947-954, 2010.

9. Jung JS, Kim DH and Kim HS: Ginsenoside Rh1 suppresses inducible nitric oxide synthase gene expression in IFN- $\gamma$ stimulated microglia via modulation of JAK/STAT and ERK signaling pathways. Biochem Biophys Res Commun 397: 323-328, 2010.

10. Wang CZ, Aung HH, Ni M, et al: Red American ginseng: ginsenoside constituents and antiproliferative activities of heatprocessed Panax quinquefolius roots. Planta Med 73: 669-674, 2007.

11. Bielack SS, Kempf-Bielack B, Delling G, et al: Prognostic factors in high-grade osteosarcoma of the extremities or trunk: an analysis of 1,702 patients treated on Neoadjuvant Cooperative Osteosarcoma Study Group protocols. J Clin Oncol 20: 776-790, 2002.

12. Torres K and Horwitz SB: Mechanisms of Taxol-induced cell death are concentration dependent. Cancer Res 58: 3620-3626, 1998.

13. Gamet-Payrastre L, Li P, Lumeau S, et al: Sulforaphane, a naturally occurring isothiocyanate, induces cell cycle arrest and apoptosis in HT29 human colon cancer cells. Cancer Res 60: 1426-1433, 2000.

14. Hindley C and Philpott A: Co-ordination of cell cycle and differentiation in the developing nervous system. Biochem J 444: 375-382, 2012.

15. Robertson JD and Orrenius S: Role of mitochondria in toxic cell death. Toxicology 181-182: 491-496, 2002. 
16. Zhao CQ, Jiang LS and Dai LY: Programmed cell death in intervertebral disc degeneration. Apoptosis 11: 2079-2088, 2006.

17. Martinou JC and Youle RJ: Mitochondria in apoptosis: Bcl-2 family members and mitochondrial dynamics. Dev Cell 21: 92-101, 2011.

18. Autret A and Martin SJ: Bcl-2 family proteins and mitochondrial fission/fusion dynamics. Cell Mol Life Sci 67: 1599-1606, 2010.

19. Mukherjee AK, Basu S, Sarkar N and Ghosh AC: Advances in cancer therapy with plant based natural products. Curr Med Chem 8: 1467-1486, 2001.

20. Park HM, Kim SJ, Kim JS and Kang HS: Reactive oxygen species mediated ginsenoside Rg3- and Rh2-induced apoptosis in hepatoma cells through mitochondrial signaling pathways. Food Chem Toxicol 50: 2736-2741, 2012.

21. Jiang JW, Chen XM, Chen XH and Zheng SS: Ginsenoside Rg3 inhibits hepatocellular carcinoma growth via intrinsic apoptotic pathway. World J Gastroenterol 17: 3605-3613, 2011.

22. Stewart ZA, Westfall MD and Pietenpol JA: Cell-cycle dysregulation and anticancer therapy. Trends Pharmacol Sci 24: 139-145, 2003.

23. Haupt S, Berger M, Goldberg Z and Haupt Y: Apoptosis - the p53 network. J Cell Sci 116: 4077-4085, 2003.

24. Kerr JF, Wyllie AH and Currie AR: Apoptosis: a basic biological phenomenon with wide-ranging implications in tissue kinetics. Br J Cancer 26: 239-257, 1972.
25. Luo X, Budihardjo I, Zou H, Slaughter C and Wang X: Bid, a $\mathrm{Bcl} 2$ interacting protein, mediates cytochrome $c$ release from mitochondria in response to activation of cell surface death receptors. Cell 94: 481-490, 1998.

26. Li QF, Shi SL, Liu QR, et al: Anticancer effects of ginsenoside Rg1, cinnamic acid, and tanshinone IIA in osteosarcoma MG-63 cells: nuclear matrix downregulation and cytoplasmic trafficking of nucleophosmin. Int $\mathrm{J}$ Biochem Cell Biol 40: 1918-1929, 2008.

27. Rello S, Stockert JC, Moreno V, et al: Morphological criteria to distinguish cell death induced by apoptotic and necrotic treatments. Apoptosis 10: 201-208, 2005.

28. Autret A and Martin SJ: Emerging role for members of the Bcl-2 family in mitochondrial morphogenesis. Mol Cell 36: 355-363, 2009.

29. Rannou F, Lee TS, Zhou RH, et al: Intervertebral disc degeneration: the role of the mitochondrial pathway in annulus fibrosus cell apoptosis induced by overload. Am J Pathol 164: 915-924, 2004.

30. Fribley A, Zhang K and Kaufman RJ: Regulation of apoptosis by the unfolded protein response. Methods Mol Biol F559: 191-204, 2009. 